Published in "Biochimica et Biophysica Acta (BBA)

- Gene Regulatory Mechanisms 1849(11): 1329-1339, 2015"

which should be cited to refer to this work.

\title{
PKA-chromatin association at stress responsive target genes from Saccharomyces cerevisiae
}

\author{
Leticia Baccarini ${ }^{\mathrm{a}}$, Fernando Martínez-Montañés ${ }^{\mathrm{c}}$, Silvia Rossi ${ }^{\mathrm{a}}$, Markus Proft ${ }^{\mathrm{b}}$, Paula Portela ${ }^{\mathrm{a}, *}$ \\ a Departamento de Química Biológica, Facultad de Ciencias Exactas y Naturales, IQUIBICEN-CONICET, Universidad de Buenos Aires, Buenos Aires, Argentina \\ ${ }^{\mathrm{b}}$ Instituto de Biomedicina CSIC, Valencia, Spain

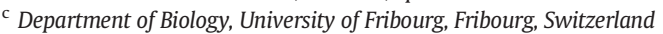

\begin{abstract}
Gene expression regulation by intracellular stimulus-activated protein kinases is essential for cell adaptation to environmental changes. There are three PKA catalytic subunits in Saccharomyces cerevisiae: Tpk1, Tpk2, and Tpk3 and one regulatory subunit: Bcy1. Previously, it has been demonstrated that Tpk1 and Tpk2 are associated with coding regions and promoters of target genes in a carbon source and oxidative stress dependent manner. Here we studied five genes, ALD6, SED1, HSP42, RPS29B, and RPL1B whose expression is regulated by saline stress. We found that PKA catalytic and regulatory subunits are associated with both coding regions and promoters of the analyzed genes in a stress dependent manner. Tpk1 and Tpk2 recruitment was completely abolished in catalytic inactive mutants. $B C Y 1$ deletion changed the binding kinetic to chromatin of each Tpk isoform and this strain displayed a deregulated gene expression in response to osmotic stress. In addition, yeast mutants with high PKA activity exhibit sustained association to target genes of chromatin-remodeling complexes such as Snf2-catalytic subunit of the SWI/SNF complex and Arp8-component of INO80 complex, leading to upregulation of gene expression during osmotic stress. Tpk1 accumulation in the nucleus was stimulated upon osmotic stress, while the nuclear localization of Tpk2 and Bcy1 showed no change. We found that each PKA subunit is transported into the nucleus by a different $\beta$-karyopherin pathway. Moreover, $\beta$-karyopherin mutant strains abolished the chromatin association of Tpk1 or Tpk2, suggesting that nuclear localization of PKA catalytic subunits is required for its association to target genes and properly gene expression.
\end{abstract}

\section{Introduction}

Signal transduction pathways play an important function in proper cellular response against extracellular injuries. Cellular adaptation to environmental changes involves a strict regulation of gene expression. In eukaryotic cells, stress-activated protein kinases (SAPKs) have an essential role in adaptation to an extracellular stimulus [1].

In response to stress or nutritional conditions, protein kinases can regulate gene expression by association to chromatin both in promoters and/or actively transcribed regions and further phosphorylation of transcription factors, histones, chromatin-modifying complexes, and/ or the transcription machinery. In Saccharomyces cerevisiae protein kinases such as Hog1 [2,3], Fus3, Kss1, Tor1 [4], Sch9 [5], and PKA [6,7] as well as Schizosaccharomyces pombe Sty1 [8] have been described as chromatin-associated kinases.

Yeast PKA acts positively on phenotypes associated with rapid fermentative growth and negatively on phenotypes associated with

* Corresponding author at: Laboratorio de Biología Molecular y Transducción de Señales, Departamento de Química Biológica, Facultad de Ciencias Exactas y Naturales UBA, Ciudad Universitaria Pabellón II Piso 4, Buenos Aires, Argentina.

E-mail address: pportela@qb.fcen.uba.ar (P. Portela). slow respiratory growth or stationary phase [9]. There are three PKA catalytic subunits in S. cerevisiae: Tpk1, Tpk2, and Tpk3 and one regulatory subunit: Bcy1 [10,11].

PKA signaling pathway controls the expression of several genes, both positively and negatively. PKA is known to inhibit Rim15, a positive regulator of the transcription factor Gis1, which induces genes required for postdiauxic growth [12], PKA also inhibits the transcription factors Msn2/4, which induces general stress responsive gene expression [13]. Nuclear localization of Msn2/4 is controlled by PKA [14]. On the other hand, it has been described that PKA negatively regulates the activity of the RNA polymerase II machinery by direct phosphorylation of Srb9, a component of the Srb Mediator complex [15], and also promoting the accumulation of inactive RNA Pol II at ribosome biogenesis genes [16]. Recently, it has been described that in response to glucose, PKA phosphorylates the transcription factor Rgt1 promoting its dissociation from HXT promoters, resulting in the upregulation of HXT genes [17]. Finally, PKA controls FLO11 gene expression not only positively by Tpk2 [18,19], but also negatively via Tpk1 through the kinase Yak1 [20].

Genome wide ChIP-on-Chip experiments in S. cerevisiae revealed that Tpk1 and Tpk2 interacted with chromatin. Tpk1 was found physically associated with genes that are actively transcribed during glucose growth or under low oxidative stress. Tpk2 was found mainly associated 
with the promoter regions of ribosomal protein genes under oxidative stress. Occupancy of the Tpk3 subunit was not detected due its low protein expression level [6].

Subcellular localization of PKA subunits is strongly regulated by nutrient availability and stress conditions. In exponentially glucosegrowing cells, Bcy1 and Tpk2 localization is mainly nuclear, whereas Tpk1 and Tpk3 show a nucleus-cytoplasmic distribution [21,22]. Cell in stationary phase of growth or stressed cells show re-localization of all PKA subunits towards the cytoplasm, where Tpk2 and Tpk3, in particular, are accumulated in RNP (RiboNucleoProtein) granules [21,23].

The traffic of RNA and protein between nucleus and cytoplasm is mediated by specialized carriers that translocate these macromolecules across the nuclear membrane. The budding yeast $S$. cerevisiae has 14 members of the $\beta$-karyopherin family which are classified into importins and exportins, depending on whether they transport the cargo in or out of the nucleus. Most $\beta$-karyopherins directly bind the NLS (Nuclear Localization Signals) of their cargos. The importin- $\beta$ pathway (Kap95 in yeast) recognizes the classical NLS through the adaptor protein importin- $\alpha$ (Kap60 in yeast). The $\beta$-karyopherins Kap108, Kap120, Kap123, and Kap114 are functionally redundant and act as importins or exportins [24-26]. Not only the $\beta$-karyopherins can recognize more than one cargo and potentially also more than one NLS, but also one cargo can be recognized by more than one $\beta$-karyopherin $[27,28]$. The nuclear-cytoplasmic transport of proteins is a flexible mechanism that responds to nutrient availability and stress $[29,30]$. The $\beta$-karyopherin pathway responsible of the nuclear traffic of S. cerevisiae PKA subunits has not been previously analyzed.

Here we determined that Tpk1 accumulates in the nucleus postosmotic stress, however Tpk2 and Bcy1 did not change their nucleuscytoplasmic distribution, being preferentially nuclear. We found that each PKA subunit is actively transported into the nucleus by different $\beta$-karyopherins pathways. We found that PKA subunits associate with different gene regions in response to osmotic stress. Catalytically inactive versions of Tpk1 and Tpk2 do not associate with the analyzed gene regions. A strain with a deletion of the $B C Y 1$ gene showed a higher Tpk1 recruitment in comparison with a WT strain, and abolished the Tpk2 association with chromatin. Deregulated PKA activity, as displayed in a $b c y 1 \Delta$ strain, caused a deregulated gene expression in response to osmotic stress and an increased osmosensitivity. Yeast mutants expressing Tpk1 or Tpk2 as a sole kinase source suggest that each Tpk catalytic isoform could play different roles in the gene expression regulation in response to osmotic stress. Particularly, Tpk2 deregulated kinase activity promoted high sustained association to target genes of Snf2 and Arp8 in correlation with an up regulation of mRNA levels.

Using $\beta$-karyopherin mutant strains we observed that gene expression response to osmotic stress is dependent on the proper nuclear localization of PKA subunits and its physical interaction with chromatin. In summary, our results indicate that PKA might be involved in gene regulation by association to genomic regions in response to osmotic stress.

\section{Materials and methods}

\subsection{Yeast strains, plasmids, media and growth conditions}

Yeast strains and plasmids used in this study are described in Supplementary Tables 1 and 2 respectively. The inactive version of Tpk 1 and Tpk2 were expressed by pTD55 and pTD53 respectively [31]. Yeast strains were transformed using the lithium acetate methods [32], and transformants were selected on SD medium lacking the appropriate amino acid supplement. Epitope tagging was constructed using the cellular repair machinery to incorporate the PCR fragment into the genomic locus [33]. A fragment containing the C-terminal coding region of the corresponding gene fused to GFP or TAP epitope carrying the HIS3 selectable marker was amplified by PCR from genomic DNA using the following primers: TPK1 gene: For 5'TTGTTAAGGAAAGCCCAAAGATTT
CCCAAC3' and Rev: 5'GATTCCGACCTTGTTTGGAGC3'. TPK2 gene: For 5' ACTGCAGATTTGACAAGAAG3' and Rev: 5'GTGCGCCAGATTTTGGTGGA 3'. BCY1 gene: For 5'AGACCATGATTATTTCGGTG3' and Rev: 5'GTAGTA ACAGCAGTAGTAGA3'SNF2 gene: For 5'ACTTCAAGCGTGGCTGAATC3' and Rev: 5'CATCCCAACTCGGTTAATGG3'. ARP8 gene: For 5'CGTGATAT GAATCCCGCTCT3' and Rev: 5'TGCTTCGTTGATGTCTGCAA3' employing genomic DNA from the corresponding strain (see Table 1). DNA sequence was confirmed by sequencing and expression of tagged protein was monitored by western-blot and fluorescence accordingly. Strains were grown in rich medium containing $2 \%$ bactopeptone, $1 \%$ yeast extract, and $2 \%$ glucose (YPGlu) at $30{ }^{\circ} \mathrm{C}$. Solid media contained $2 \%$ agar.

\subsubsection{Osmotic stress, expression levels, and cell viability}

Osmotic stress was performed by addition of $0.4 \mathrm{M} \mathrm{NaCl}$. Aliquots were taken at different times and processed according to each determination. The zero time point corresponds to samples taken immediately before $\mathrm{NaCl}$ addition. Expression level analysis: crude extracts of the strains indicated on each graph were prepared according to Materials and methods, and were subjected to SDS/PAGE, Western blotting using an anti-GFP antibody (Supplementary Fig. S1A) or anti-TAP antibody (Supplementary Fig. S3A) and quantified by densitometry as described below. The expression levels of each tagged protein in different strains for each condition were expressed relative to the expression levels under normal condition. The bars represent the mean \pm SEM from two independent experiments. Cell viability: strains were grown to exponential phase at $30^{\circ} \mathrm{C}$ and treated with $0.4 \mathrm{M} \mathrm{NaCl}$ during $40 \mathrm{~min}$. Cell viability of wild type and mutant strains after $\mathrm{NaCl}$ treatment was verified by spot assay method (Supplementary Fig. S1B and Supplementary Fig. S3B).

\subsubsection{Osmosensitivity assay}

Serial dilutions of exponentially growing cells on YPGlu were spotted directly onto the plates containing different $\mathrm{NaCl}$ concentrations. Plates were photographed after 2 days at $30^{\circ} \mathrm{C}$.

\subsubsection{Energy dependent import assay}

The protocol was performed as described [34,35]. Cells were grown on YPGlu until exponential growth at $30^{\circ} \mathrm{C}$. Cells were harvested at room temperature by pelleting at $3500 \times \mathrm{g}$. Cell pellets were washed with and resuspended in $1 \mathrm{ml}$ of $10 \mathrm{mM}$ sodium azide, $10 \mathrm{mM} \mathrm{2-}$ deoxy-o-glucose in glucose-free YP medium and incubated for $60 \mathrm{~min}$ at $30{ }^{\circ} \mathrm{C}$ to allow nucleocytoplasmic equilibration of GFP-tagged PKA subunits fluorescence. After $60 \mathrm{~min}$, the cultures were pelleted and resuspended in $30{ }^{\circ} \mathrm{C}$ pre warmed YPGlu fresh medium and incubated for $60 \mathrm{~min}$. Aliquots of each culture were processed at indicated times for fluorescence microscopy.

\subsubsection{Doxycycline treatment}

Glucose exponentially growing cells expressing Bcy1-GFP, Tpk1-GFP or Tpk2-GFP in wild type or tet0::KAP95 genetic background (described in SupplementaryTable 1) were treated or not with $5 \mu \mathrm{g} / \mathrm{ml}$ doxycycline for $6 \mathrm{~h}$. The lack of expression of gene KAP95 was assayed by the sensitivity to zymolyase treatment assay: cells were resuspended in water and digested with $1 \mathrm{mg} / \mathrm{ml}$ zymolyase $20 \mathrm{~T}$ and the OD $600 \mathrm{~nm}$ was determined; the repression of gene KAP95 was verified by Northern blot.

\subsection{SDS-PAGE and Western blot}

The pelleted cells were disrupted in an appropriate buffer with glass beads and the lysate was clarified. Samples of crude extracts were separated by $10 \%$ SDS-PAGE. The gels were blotted onto nitrocellulose membranes. Blots were probed with anti-TAP (Open Biosynthesis) or anti-GFP (Santa Cruz antibodies). The blots were developed with Chemiluminescence Luminol reagent, and immunoreactive bands were visualized by autoradiography and analyzed by digital imaging using Bio-Imaging Analyzer Bas-1800II. To quantify Western blots, 

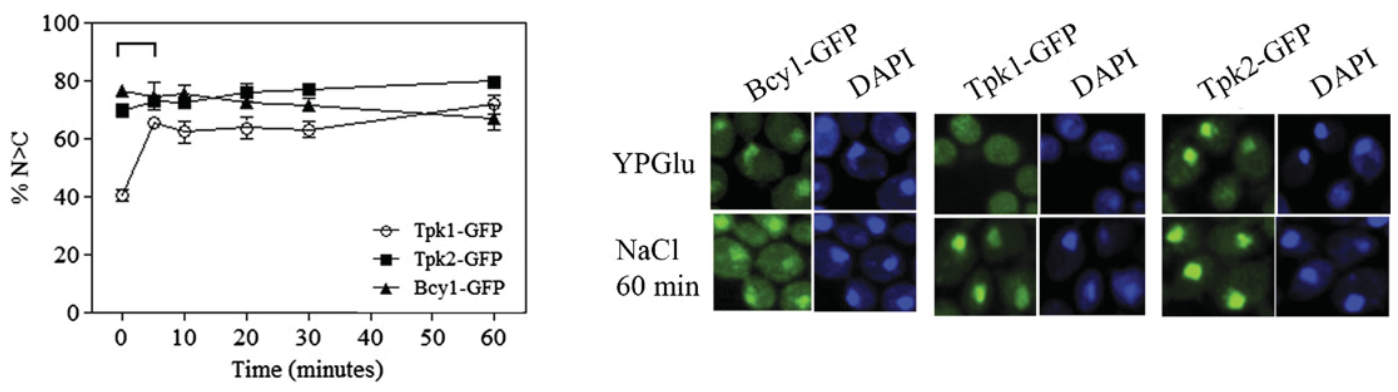

Fig. 1. Kinetics of subcellular localization of PKA subunits in response to osmotic stress. Cells expressing Bcy1-GFP, Tpk1-GFP or Tpk2-GFP were grown until exponential phase and stressed with $0.4 \mathrm{M} \mathrm{NaCl}$. Aliquots of cultures pre- and post-different time of $\mathrm{NaCl}$ addition were processed; nuclei were stained with DAPI and visualized by fluorescence microscopy. Left graph shows a quantification of localization pattern: $\mathrm{N}>\mathrm{C}$ cells with nuclear fluorescence stronger than cytoplasmic fluorescence. Each value represents the mean $\pm \mathrm{SEM}$, from two independent experiments (brackets denote significant differences $P<0.05$ Time 0 versus Time 10 min Tpk1-GFP). The right panels show representative images.

short exposures were scanned and quantified using Image Gauge 3.12 software.

\subsection{Fluorescence microscopy}

Cells used for fluorescence microscopy were grown to early logarithmic phase, fixed with $7.4 \%$ formaldehyde, washed with PBS buffer, and re-suspended in $0.05 \%$ Triton plus $1 \mu \mathrm{g} \mathrm{ml} \mathrm{m}^{-1}$ DAPI (4,6-diamidino-2phenylindol) for 30 min to stain the nuclei. Microscopy was performed using an epi-fluorescence microscope (Nikon Eclipse E600W). The images were processed using ImageJ (National Institute of Health) and Adobe Photoshop CS2 software.

\subsection{ChIP assays}

ChIP was performed as described previously [36]. Briefly, yeast cultures were grown to exponential phase. Aliquots of the culture were exposed to osmotic-stress treatment $(0.4 \mathrm{M} \mathrm{NaCl})$ for various different times. For cross-linking, yeast cells were treated with $1 \%$ formaldehyde for $20 \mathrm{~min}$ at room temperature. Immunoprecipitation was performed with magnetic Dynabeads (Invitrogen).

From the ChIP-on-Chip raw data published by Pokholok et al., 2006, we selected the 5 gene regions with highest binding by Tpk1/2: open reading frame regions: ALD6: chromosome 16, position 433,366; SED1: chromosome 4, position 601,413; HSP42: chromosome 4, position 807,396 . The intragenic regions were selected corresponding to chromosome 4, position 341,449 assigned as RPS29B promoter and chromosome 7, position 254,309 assigned as RPL1B promoter. The oligonucleotides used to amplify open reading frame regions were: ALD6/YPL061W (+ 730 + 842) For 5'AGCTGGCTTTTACCGGTTCT3' Rev: 5' ACCAAATGGGCGGACTTAC3'; SED1/YDR077W (+827+921) For 5' GGGCACTACCACCAAAGAAA3' Rev: 5'AGAGGATGAAACTGGGACGA3'; HSP42/YDR171W $(+865+1014)$ For 5'AGAGTGGGCATTGATGAAAA3' Rev: 5'AGGCACCTTAATTTGTAGTAGAC3'. The oligonucleotides used to amplify intragenic regions were: RPS29B $(-750-603)$ For $5^{\prime}$ ATCAAC TTCTAACTCACACA3' Rev: 5'CCCCCAAGTGAAATAAATAG3'; RPL1B (-295-371) For 5'CTTCGGCCCCACAAACTC3' Rev: 5'CGCTTTCCTTGG ATCAACATAC3'.

Quantitative PCR analysis of the indicated chromosomal loci was performed using a real-time Applied Biosystems 7000 sequence detector. PCR reactions were performed using Eva Green qPCR Basic HS Mix (Biotum). Each immunoprecipitation was performed two or three times with different chromatin samples. Enrichment of immunoprecipitation is represented by $\Delta \mathrm{Ct}$ ( $\mathrm{Ct}$ value (IP sample) minus $\mathrm{Ct}$ value (input)) relative to the untagged control samples.

\subsection{Northern blot analysis}

Northern analysis was carried out as described previously [37]. Total RNA was prepared using the hot phenol method. Gene expression was analyzed using ${ }^{32}$ P-labeled PCR fragments of ALD6 $(+662+1150)$, SED1 (+643 + 977), HSP42 (+245+417), RPL1B $(+61+421)$, RPS29B $(+1+171)$ and KAP95 $(+2167+2413)$. The membranes were developed by autoradiography using a Phospholmager. The mRNA levels were quantified by densitometric analysis and normalized to the $25 \mathrm{~S}$ rRNA ribosomal RNA using ImageJ (National Institutes of Health).

\subsection{Reproducibility of the results}

All the experiments were repeated several times (indicated in each figure) using independent cultures. Results shown in Figs. 1, 2B and D, $3 \mathrm{D}$, and 4 were analyzed using repeated measures ANOVA Bonferroni post-test, $P<0.05$. Results shown in Figs. 2A, $C$ and 5 were analyzed using ANOVA-Tukey HSD, $P<0.05$.

\section{Results}

\subsection{PKA subunit localization in response to osmotic stress}

We studied the dynamic changes of PKA subunits subcellular localization during osmotic stress evoked by $\mathrm{NaCl}$ addition. Bcy1-GFP, Tpk1-GFP, and Tpk2-GFP subcellular localization was analyzed using genomically GFP-tagged strains. Exponentially growing cells in YPGlu were stressed with $0.4 \mathrm{M} \mathrm{NaCl}$ during $60 \mathrm{~min}$ and nuclei were stained with DAPI as described in Materials and methods. Cell viability and the expression levels of the tagged PKA subunits were similar after $\mathrm{NaCl}$ treatment (see Materials and methods, Supplementary Fig. S1A and B).

As previously described [20], in cells growing at exponential phase on glucose (Fig. 1, time 0 min) Bcy1 and Tpk2 show predominantly nuclear localization ( $\mathrm{N}>\mathrm{C} 76 \%$ and $70 \%$ respectively) while Tpk1 is distributed between nucleus and cytoplasm ( $\mathrm{N}>\mathrm{C} 40 \%$ ). In response to osmotic stress, Tpk2 and Bcy1 do not change their nuclear localization, while Tpk1 is mainly nuclear 5 min post-stimulus ( $\mathrm{N}>\mathrm{C} 65 \%$ ) (Fig. 1). Inhibition of protein translation by the addition of cycloheximide, previous to osmotic stress, does not affect the subcellular localization pattern of PKA subunits (results not shown). This result suggests that Tpk1 translocates from the cytoplasm to the nucleus in response to osmotic stress.

There is no information regarding the mechanism by which PKA subunits translocate to the nuclei. To begin the study of this mechanism we analyzed whether the cytoplasmic-nuclear transport of PKA subunit was an active process or not. Cell cultures expressing Bcy1-GFP, Tpk1GFP or Tpk2-GFP were grown up to exponential phase in glucose rich medium, depleted of ATP by incubation with glucose-free medium with the addition of sodium azide and 2-deoxy-D-glucose during 40 min and energetically restored by addition of fresh rich medium. Subcellular localization of PKA subunits was analyzed (Supplementary Fig. S2). Nuclear accumulation of PKA subunits decreased as time of ATP depletion progressed and was restored upon cellular energy restitution suggesting an active nuclear-cytoplasmic transport of PKA 
A

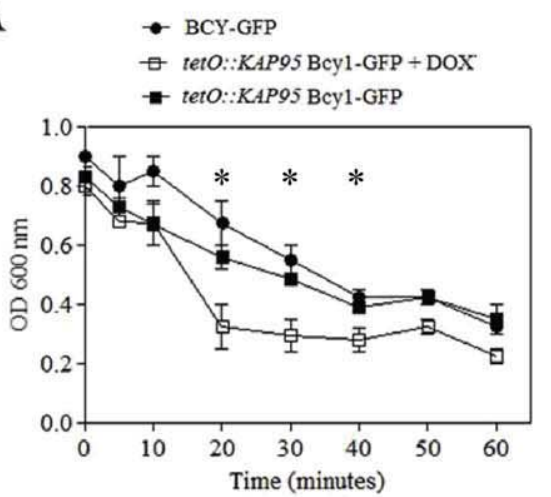

B

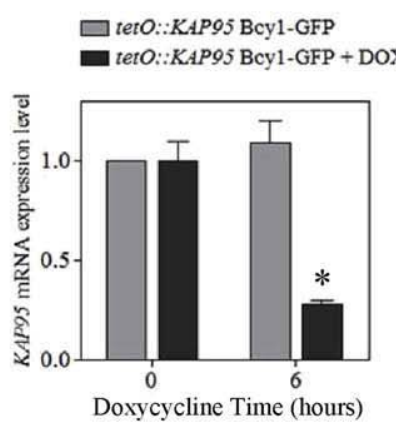

- tetO: :KAP95 Bcyl-GFP

․ tetO::KAP95 Bcy1-GFP + DOX

- tetO::KAP109 Bcy1-GFP

4. tetO::KAP109 Bcy1-GFP + DOX

- Bcy1-GFP

- Bcyl-GFP + DOX

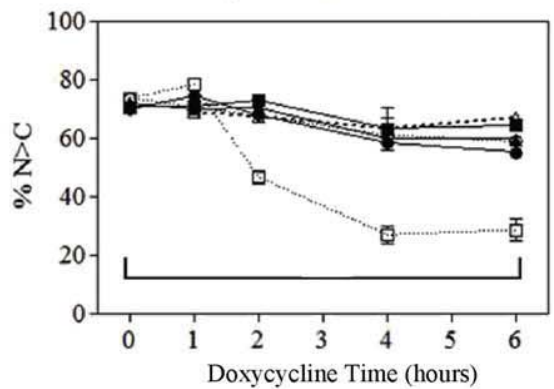

C

Bcyl-GFP

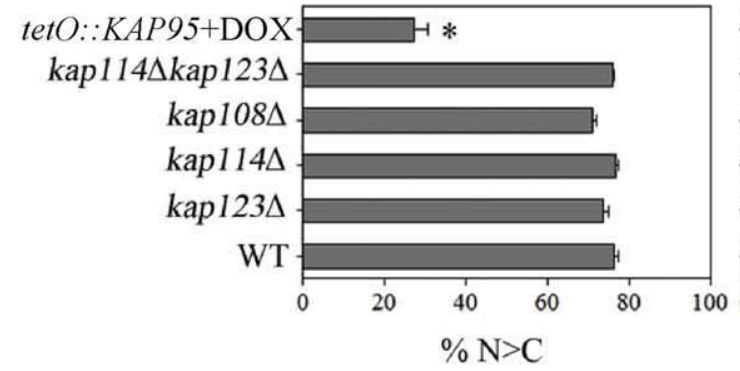

Tpk1-GFP

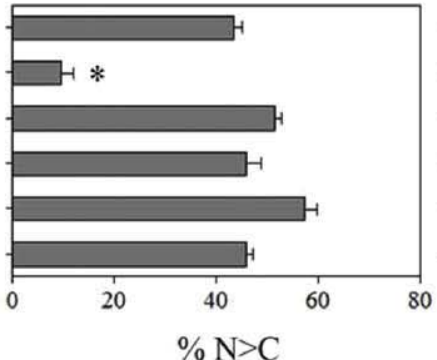

Tpk2-GFP

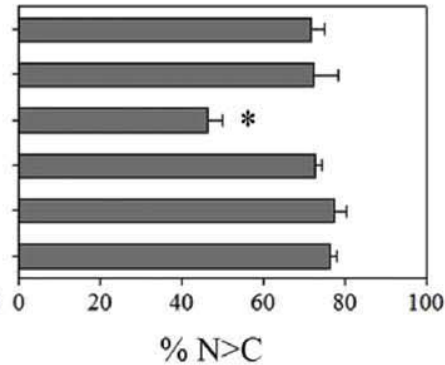

pk1-GFP DAPI

tetO::KAP95+DOX

WT

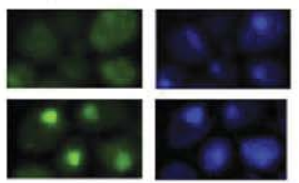

kap1144

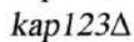

kap $114 \Delta$

kap123⿻

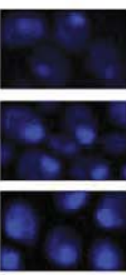

WT
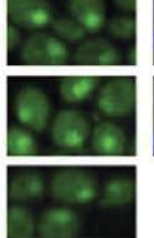
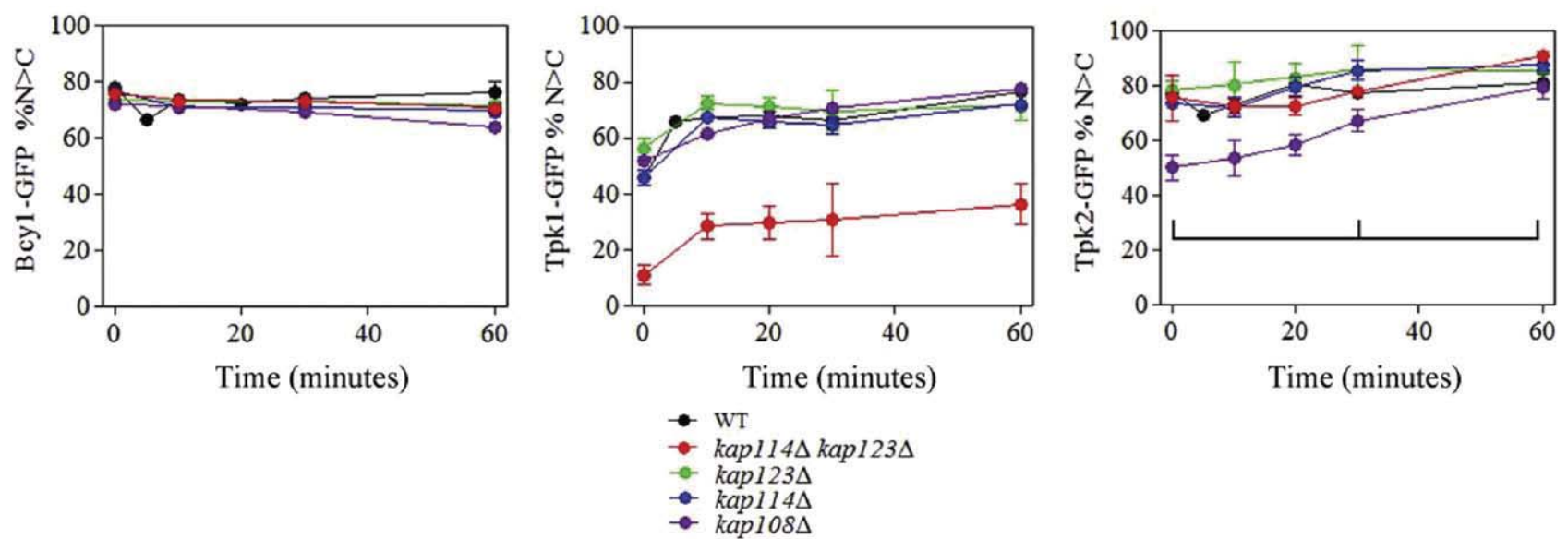

kap1084

WT

Tpk2-GFP

DAPI
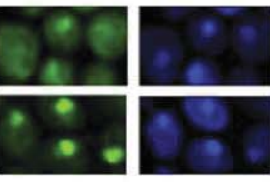

Time (minutes) 
subunits. In response to energy restitution, nuclear accumulation of Tpk1 was similar to Tpk2 and Bcy1 ( $>$ C 60\%).

In order to identify the members of the $\beta$-karyopherin family involved in the nuclear import of PKA subunits in vivo, we constructed

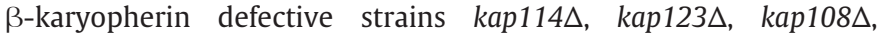

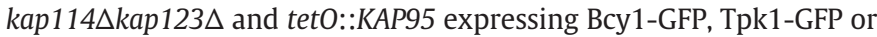
Tpk2-GFP from its chromosomal locus (Supplementary Table 1). Since the deletion of KAP95 gene is lethal a strain tet0::KAP95 was used. In this strain the KAP95 expression is inhibited by TetR repressor in the presence of doxycycline. The lack of expression of the gene KAP95 affects cell wall integrity and cells become highly sensitive to zymolyase treatment [38]. We verified the repression of KAP95 gene expression induced by doxycycline treatment both by measuring the relative hypersensitivity of this strain to zymolyase lysis as compared to control conditions (Fig. 2A left panel) and by Northern blot (Fig. 2A right panel). Fig. 2B and $C$ show that the nuclear localization of Bcy1-GFP in the tetO::KAP95 strain was highly decreased upon doxycycline treatment. The control using the Bcy1-GFP tetO::KAP109 strain indicated that the observed mislocalization of Bcy1 was a consequence of KAP95 gene repression and not due to doxycycline treatment (Fig. 2B). Fig. 2C additionally shows that nuclear localization of the Tpk1-GFP subunit was affected

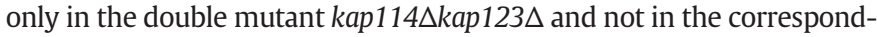
ing single mutant strains. Finally, the nuclear localization of Tpk2-GFP was partially prevented in a strain defective in Kap108. All together, these results show that Bcy1, Tpk1, and Tpk2 are ferried to the nucleus in vivo by different transport factors of the $\beta$-karyopherin family.

It has been described that $\beta$-karyopherin activities can be affected by stress [29]. In order to analyze which $\beta$-karyopherins were involved in PKA subunit nuclear accumulation in response to osmotic stress we assessed the distribution of each PKA subunit, Bcy1-GFP, Tpk1-GFP, and Tpk2-GFP, in yeast cells subjected to osmotic stress (Fig. 2D). PKA subunits expression levels and cell viability were similar before and after stress treatment in $\beta$-karyopherin mutant strains (Supplementary Fig. S1A and B) except for the strain tetO::KAP95 which was omitted from this analysis since cellular viability was severely affected (data not shown). Bcy1-GFP did not modify its nuclear accumulation in the mutant strains assayed in re-

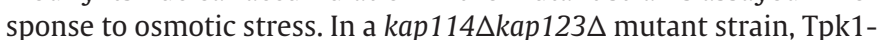
GFP showed a very slight increment in nuclear localization with the $0.4 \mathrm{M} \mathrm{NaCl}$ treatment (Fig. 2D). However, Tpk2-GFP reached a nuclear accumulation similar to WT in kap108 $\Delta$ strain post- 60 min of $\mathrm{NaCl}$ addition. The results indicate that upon osmotic stress, Tpk1 transport to the nucleus is regulated by the combined activity of Kap114 and Kap123 whereas Kap108 has not a major role in Tpk2 import to the nucleus after $40-60$ min of osmotic stress. Future studies will have to determine whether an alternative $\beta$-karyopherin pathway is involved in Tpk2 nuclear accumulation in response to osmotic stress.

\subsection{Role of PKA activity on gene expression and cellular survival in response to osmostress}

Taking into account the previous results which showed that saline stress increased Tpk1 nuclear localization we wondered whether this change in subcellular distribution could have a role in the gene expression of yeast to saline stress. To study this possibility, we chose to analyze genes which have been identified in genomic arrays as responsive to $\mathrm{NaCl}[39,40]$. It has already been demonstrated that Tpk1 and Tpk2 are recruited to these locus under fermentative metabolism or oxidative stress suggesting a role of PKA in gene expression regulation during stress [6].

We measured the mRNA levels of ALD6, SED1, HSP42, RPS29B, and $R P L 1 B$ genes during osmotic stress, in wild type and $b c y 1 \triangle$ strains to assess the effect of a deregulated PKA activity on their expression (Fig. 3A). In the bcy1 $\triangle$ strain, ALD6 mRNA level increased at $5 \mathrm{~min}$ post-stress, like in the WT strain, but the increase was not transient and the levels remained high. SED1 gene expression was transiently activated with a maximum at 5 min post-stress in wild type strain; however in bcy1 $\triangle$ strain SED1 mRNA levels were upregulated later than in WT. Expression levels of HSP42, RPS29B and RPL1B mRNA showed a strong down regulation during osmostress in the wild type strain. The loss of Bcy1 function promoted an upregulation of HSP42 and RPS29B genes in a transient or constant manner respectively. Finally, RPL1B showed a low and sustained expression in $b c y 1 \Delta$ strain during stress. The results indicate that PKA activity modulates the gene expression kinetics of these genes in response to osmotic stress.

The role of PKA activity was also assessed on cell adaptation to osmotic stress. Serial dilution aliquots of bcy $1 \Delta$ and wild type strains grown up to exponential phase in YPGlu were loaded on plates containing different concentrations of $\mathrm{NaCl}$. As previously described [41], the bcy $1 \Delta$ strain showed hypersensitivity to osmotic stress (Fig. 3B) indicating that a deregulated PKA activity is deleterious to cell survival upon osmotic stress.

Since a high PKA activity promoted the induction of its target genes, we examined the individual contribution of Tpk1 or Tpk2 catalytic isoforms to the regulation of expression of osmostress genes (Fig. 3C). Expression of ALD6 and RPS29B genes was analyzed in strains expressing only Tpk1 or Tpk2 in a bcy1 $\Delta$ background. In a strain with deregulated Tpk1 activity (Tpk1tpk2 $\Delta t p k 3 \Delta b c y 1 \Delta$ ) there was a strong reduction in both genes analyzed in response to osmotic stress. The expression analysis in cells harboring deregulated Tpk2 activity (Tpk2tpk1 $\Delta t p k 3 \Delta b c y 1 \Delta$ ) showed that ALD6 gene expression was transiently activated, like in wild type cells, although with two differences: a delay in the activation time and a higher up regulated activity after the maximum level was attained at 10 min post-osmotic stress. A strong effect on RPS29B gene was observed, the deregulated Tpk2 activity, in clear difference with deregulated Tpk1, promoted a rapid and sustained gene upregulation in response to osmostress. These results suggest that each Tpk catalytic isoform could play different roles in the gene expression regulation in response to osmotic stress.

Considering that deregulated PKA activity increased osmostress gene expression, we tested if the recruitment of chromatin remodelers changed in osmo-responsive genes by high PKA activity. Snf2, the catalytic subunit of the SWI/SNF chromatin remodeling complex has been shown to be recruited to osmotically inducible genes [42]. Arp8, component of INO80 chromatin-remodeling enzyme complexes, has an activating function for transcription [43], but also seems to be relevant for efficient downregulation of gene expression under stress conditions

Fig. 2. (A) Validation of KAP95 mutant cells. Left panel, cell integrity defect in tetO::KAP95 mutant cells. Glucose exponentially growing cells from Bcy1-GFP and tetO::KAP95 Bcy1-GFP strains were treated (+DOX) or not with $5 \mu \mathrm{g} / \mathrm{ml}$ doxycycline; after $6 \mathrm{~h}$ cells were resuspended in water, digested with $1 \mathrm{mg} / \mathrm{ml}$ zymolyase $20 \mathrm{~T}$ and OD $600 \mathrm{~nm}$ was determined. Values represent the mean \pm SEM of three independent experiments. Right panel, KAP95 mRNA expression level was determined by Northern-Blot on aliquots of cultures before and after treatment with $5 \mu \mathrm{g} / \mathrm{ml}$ doxycycline during $6 \mathrm{~h}$. Values represent the mean $\pm \mathrm{SEM}$ of two independent experiments ( $\left.{ }^{*} P<0.005\right)$. (B) Bcy1-GFP localization in tetO::KAP95 strain. TetO::KAP95 Bcy1-GFP, tetO::KAP109 Bcy1-GFP (as a control) and Bcy1-GFP strains were treated or not with $5 \mu \mathrm{g} / \mathrm{ml}$ doxycycline for up to $6 \mathrm{~h}$. At different times after doxycyline additions Bcy1-GFP subcellular localization was analyzed as described in Materials and methods. Values represent the mean \pm SEM of three independent experiments. 300 cells were counted in each experiment (brackets denote significant differences $P<0.005$ Time 0 versus Time $6 \mathrm{~h}$ ). (C) Analysis of $\beta$-karyopherins involved in in vivo nuclear import of PKA subunits. Wild type and $\beta$ karyopherin mutant strains expressing Tpk1-GFP, Tpk2-GFP or Bcy1-GFP were grown until exponential phase at $30{ }^{\circ} \mathrm{C}$ on YPGlu. Cells were fixed with formaldehyde, nuclei stained with DAPI and location analyzed by fluorescence microscopy. The strain tetO::KAP95 was pre-incubated with doxycycline during $4 \mathrm{~h}$ as described in Materials and methods. The graph indicates the percentage of cells with nuclear localization $\mathrm{N}>\mathrm{C}$. 100 cells were counted for each determination. Values represent the mean \pm SEM of three independent experiments $\left({ }^{*} P<0.005\right)$. (D) Kinetics of nuclear-cytoplasmatic transport of PKA subunits in response to osmotic stress. Strains used in C (except tetO::KAP95) were grown to exponential phase on YPGlu and osmostressed by the addition of $0.4 \mathrm{M} \mathrm{NaCl}$. Aliquots were taken at the indicated times and subcellular localization was determined as described before. Values represent the mean \pm SEM of four independent experiments (brackets denote significant differences $P<0.005$ Time 0 versus Time 30 min or Time 60 min kap108 ). 
A
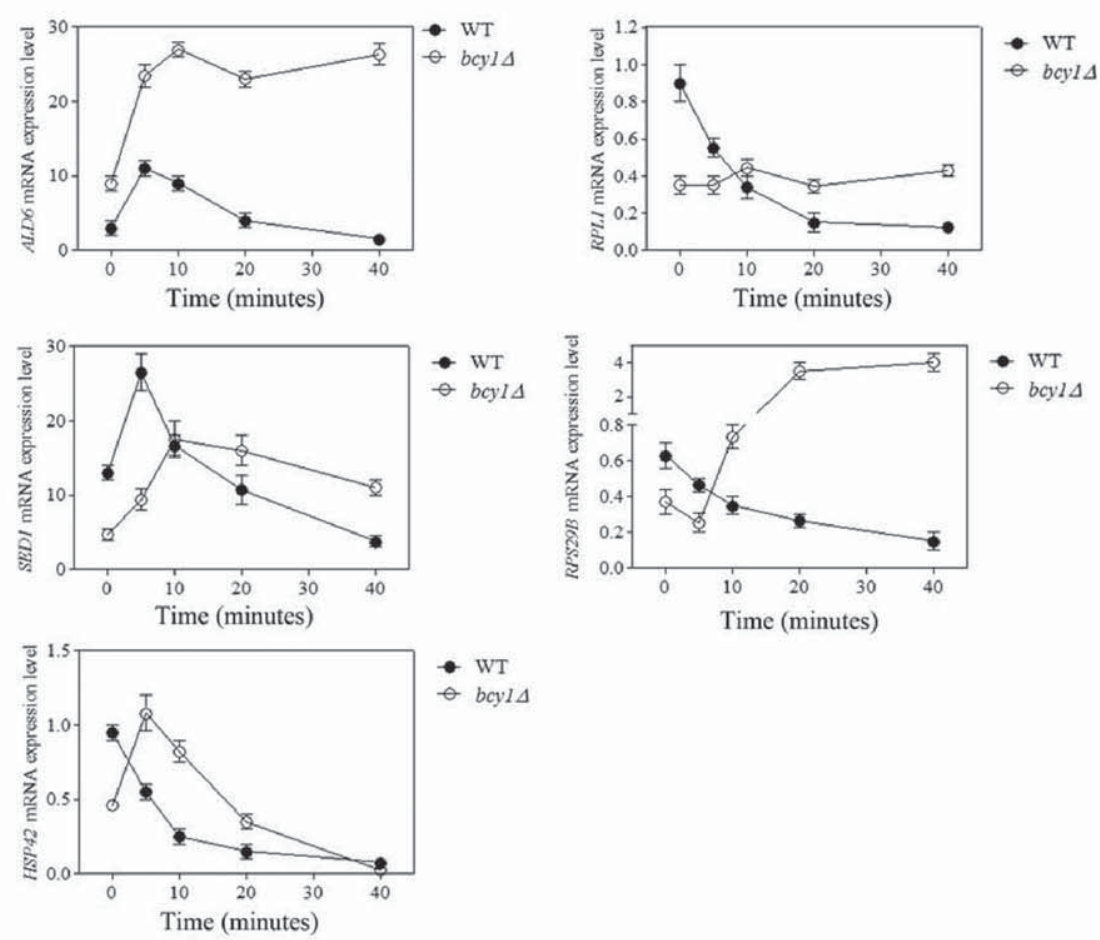

- wT

o byls

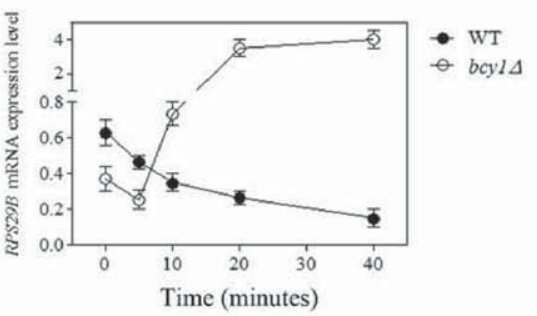

B

YPGlu

$0.5 \mathrm{M} \mathrm{NaCl}$

$1 \mathrm{M} \mathrm{NaCl}$
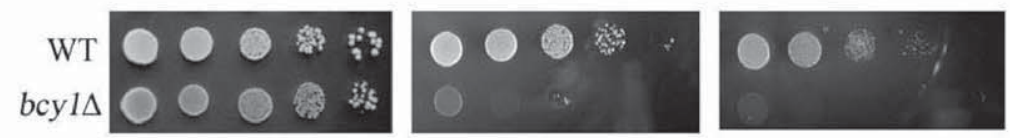

C
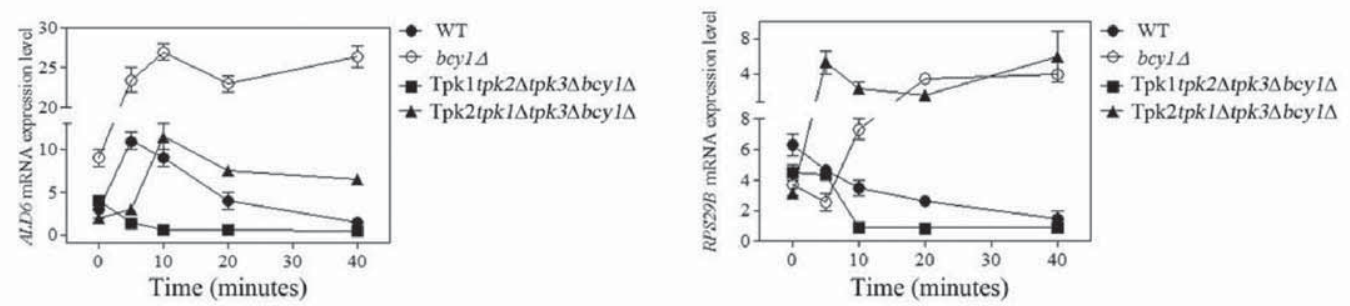

D
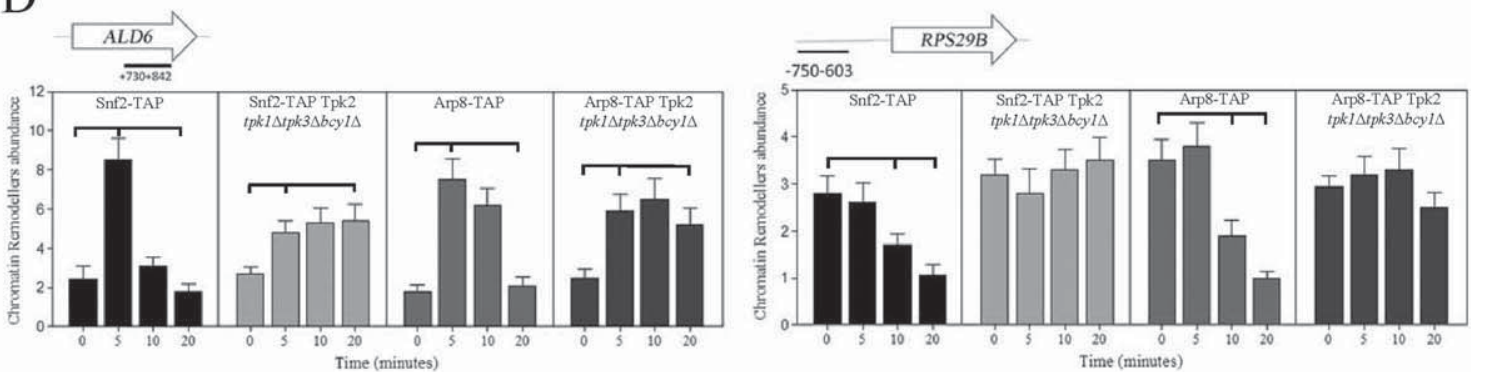

Fig. 3. (A) Analysis of PKA role on gene expression. The mRNA expression level was determined by Northern-Blot on aliquots of cultures before and after $0.4 \mathrm{M}$ NaCl addition as indicated. The strains used were wild type (WT) and $b c y 1 \Delta$. The mRNA levels were quantified by densitometric analysis and normalized to the $25 \mathrm{~S}$ rRNA ribosomal. (B) Osmosensitivity of yeast mutant $b c y 1 \Delta$ and wild type strains. The osmosensitivity was determined by spot assay. Serial dilutions (1/10) of the wild type and $b c y 1 \Delta$ strains were spotted on plates containing 0.5 or $1 \mathrm{M} \mathrm{NaCl}$ and grown for $48 \mathrm{~h}$ (see Materials and methods). (C) Analysis of the role of Tpk 1 and Tpk2 on gene expression. The mRNA expression level for ALD6 and RPS29B genes was analyzed as described in (A) using the strains Tpk1tpk2 $\Delta t p k 3 \Delta b c y 1 \Delta$ and Tpk2tpk1 $t p k 3 \Delta b c y 1 \Delta$. The values of WT and $b c y 1 \Delta$ were re-plotted from Fig. $3 A$ for comparison. (D) Kinetic ChIP analysis of chromatin remodeling complexes association in response to osmotic stress. Exponentially growing yeast cells expressing Snf2-TAP, Snf2-TAP Tpk2tpk1 $\Delta t p k 3 \Delta b c y 1 \Delta$, Arp8-TAP and Arp8-TAP Tpk2tpk1 1 tpk3 $\Delta b c y 1 \Delta$ were used. ChIP analysis of indicated protein association to the ALD6 and RPS29B chromatin regions were performed before and after different time points of osmostress $(0.4 \mathrm{M} \mathrm{NaCl})$. Fold IP was calculated as reported previously. Each bar represents the mean \pm SEM, from two independent experiments (brackets denote significant differences $P<0.005$ ALD6 gene Time 5 min versus Time 0 and 20 min Snf2-TAP and Arp8-TAP; Time 0 min versus Time 5 and 20 min Snf2-TAP Tpk2tpk1 $\Delta$ tpk3 $\Delta b c y 1 \Delta$ and Arp8-TAP and Arp8-TAP Tpk2tpk1 $\Delta$ tpk3 $\Delta b c y 1 \Delta$. RPS29B gene Time 0 min versus Time 10 and 20 min Snf2-TAP and Arp8-TAP). 
[44]. We therefore analyzed the kinetic recruitment of the remodeler subunits Snf 2 and Arp8 to the ALD6 coding and the RPS29B promoter regions in wild type cells and cells expressing deregulated PKA activity in response to osmostress (Fig. 3D). In order to do this, we constructed strains expressing Snf2-TAP or Arp8-TAP in cells expressing deregulated
PKA activity Tpk2tpk1 $1 t p k 3 \Delta b c y 1 \Delta$. Wild type and mutant strains were treated with $0.4 \mathrm{M} \mathrm{NaCl}$ and used in ChIP assays. No change in the expression levels of Arp8-TAP and Snf2-TAP proteins expressed from wild type or from a Tpk2tpk1 $1 \mathrm{tpk} 3 \Delta b c y 1 \Delta$ background was observed after osmotic stress as verified by Western blot (Supplementary

A

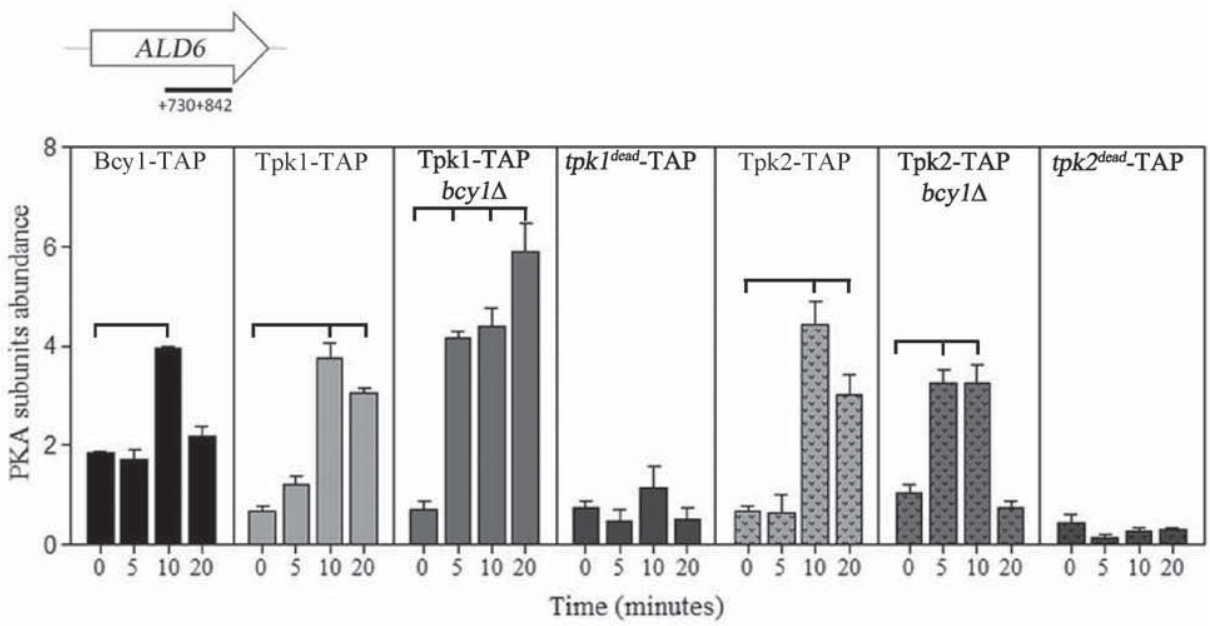

B
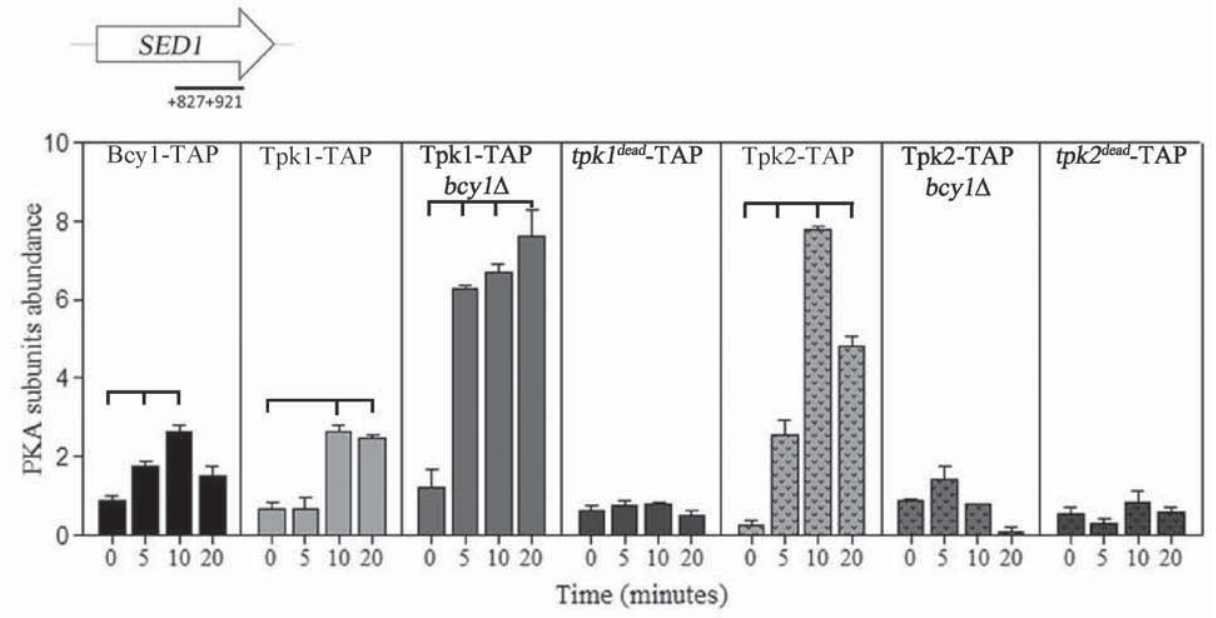

$\mathrm{C}$

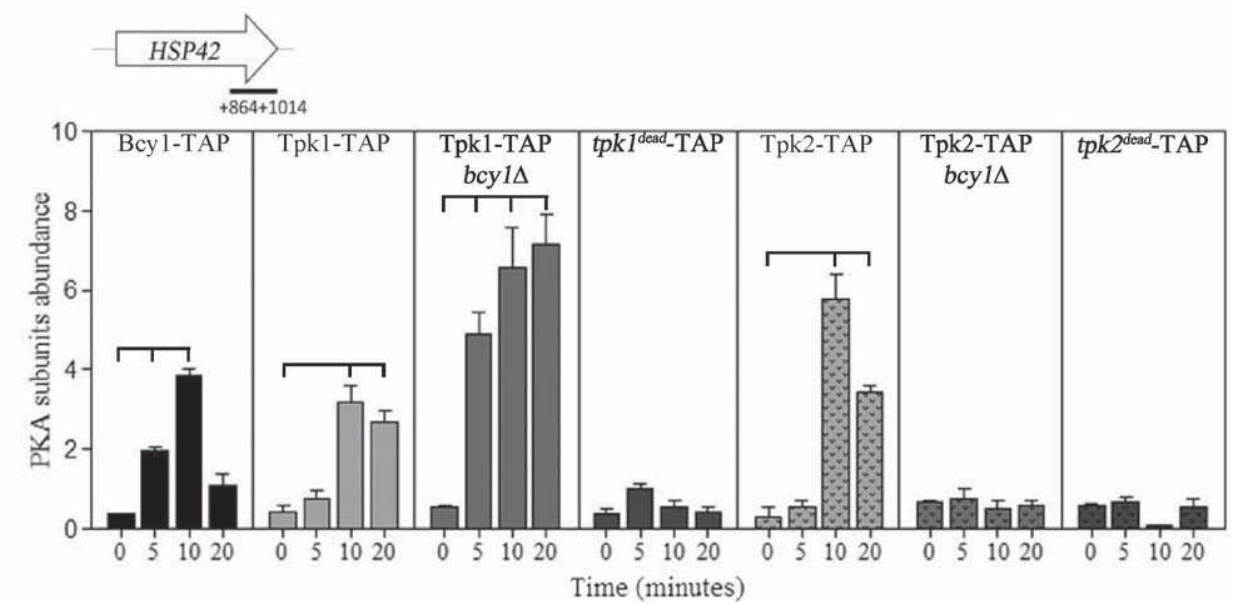

Fig. 4. Kinetic analysis of PKA subunits recruitment to the open reading frame regions and the promoter regions in response to osmotic stress in vivo. Exponentially growing yeast cells expressing Tpk1-TAP, Tpk2-TAP, Bcy1-TAP, tpk1 ${ }^{\text {dead }}$-TAP, tpk2 ${ }^{\text {dead }}$-TAP, Tpk1-TAP bcy1 1 or Tpk2-TAP bcy1 $\Delta$ were used. ChIP analysis of the indicated protein to ALD6 (A), SED1 (B), HSP42 (C), RPS29B (D) and RPL1B (E) was performed before and at the indicated times after $0.4 \mathrm{M}$ NaCl addition as osmostressor. A schematic representation of the amplified regions in the ChIP experiments is indicated. PKA subunits abundance was calculated as $\Delta \mathrm{Ct}$ (Ct value (IP sample) minus Ct value (input)) relative to the untagged control samples. Each bar represents the mean \pm SEM, from three independent experiments (brackets denote significant differences $P<0.005$ ). 
$\mathrm{D}$
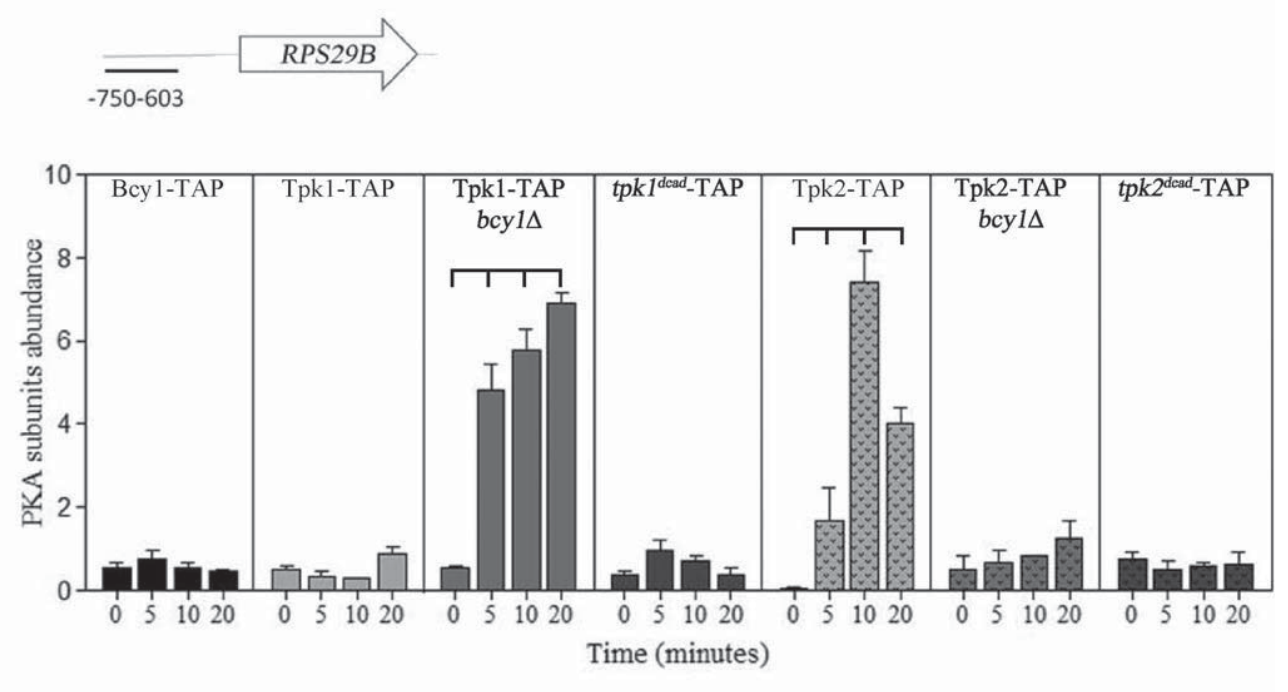

$\mathrm{E}$
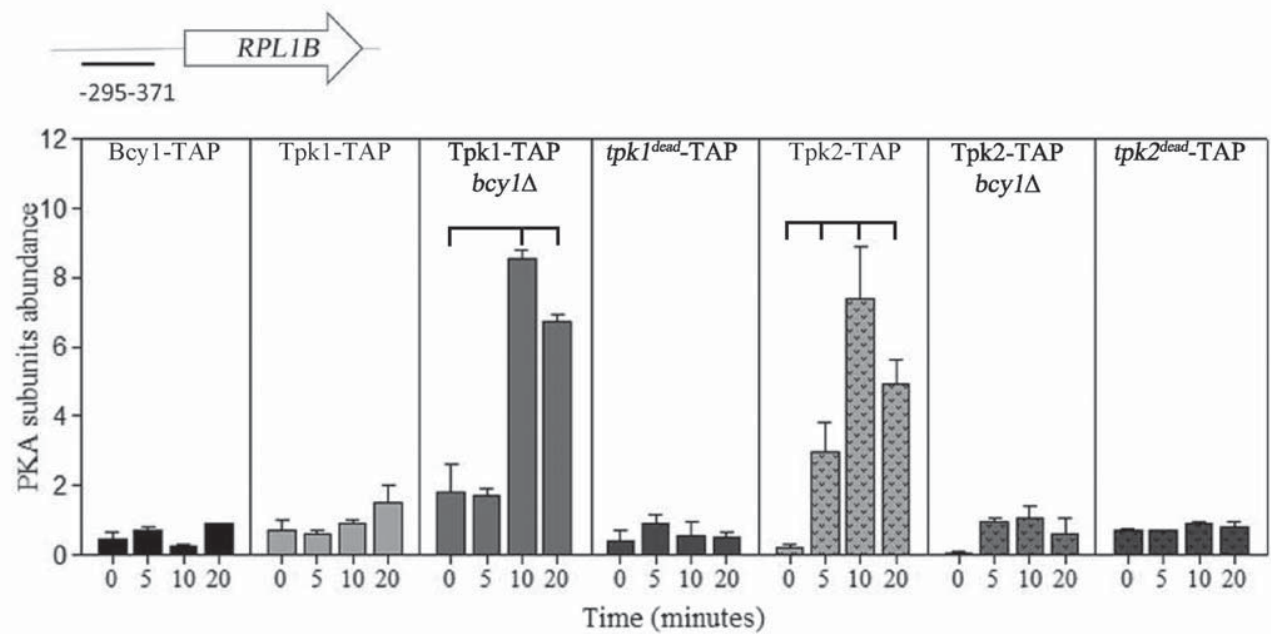

Fig. 4 (continued).

Fig. S3A) and spot assays showed that the cell viability of these strains was not affected by the $\mathrm{NaCl}$ treatment (Supplementary Fig. S3B). The results in Fig. 3D show that in response to osmotic stress Snf2 was recruited transiently to the ALD6 coding region with a maximum at 5 min, while Arp8 was transiently recruited with a maximum at 510 min post-osmotic stress. This association pattern correlated with the transiently ALD6 mRNA expression (Fig. 3C). On the contrary, in the strain with deregulated kinase activity, Tpk2tpk1 $\Delta t p k 3 \Delta b c y 1 \Delta$, a sustained association of Snf2 and Arp8 in correlation with upregulation of ALD6 mRNA expression was observed.

At the RPS29B promoter region the basal occupancy of these remodelers decreased with $\mathrm{NaCl}$ treatment in correlation with mRNA
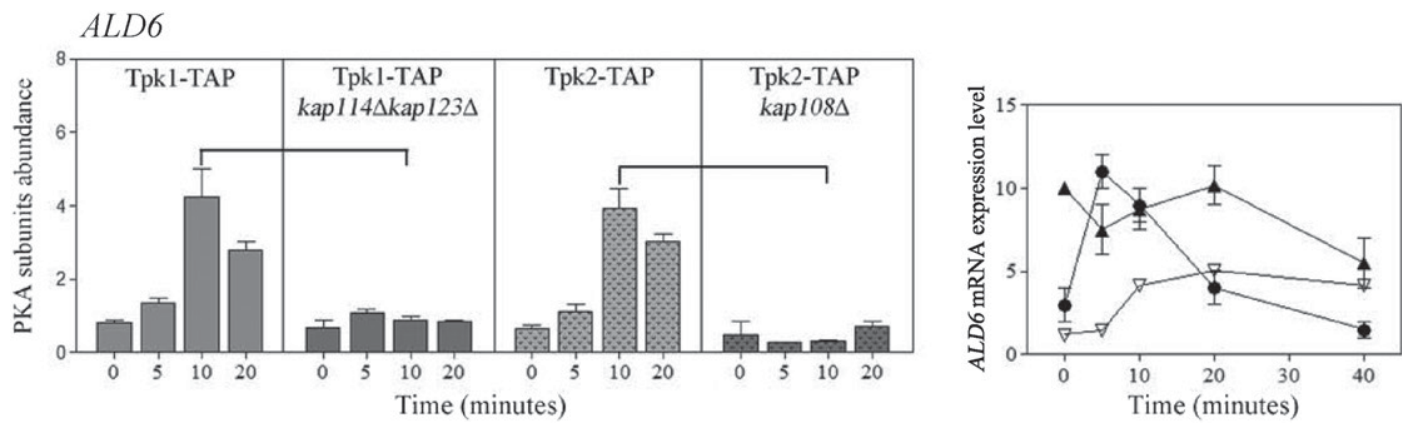

- wT

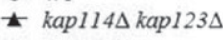

$\nabla$ kap1080

Fig. 5. Effect of nuclear mislocalization of Tpks on its association with osmoresponsive genes and gene expression. Tpk1-TAP, Tpk1-TAP kap114 $\Delta /$ kap123 $\Delta$, Tpk2-TAP and Tpk2TAP kap108 $\Delta$ strains were grown to exponential phase in YPGlu and subjected to osmotic stress by the addition of $0.4 \mathrm{M} \mathrm{NaCl}$ during $20 \mathrm{~min}$. Aliquots were taken at the indicated times and the association of PKA to the ALD6 gene was analyzed as described in Materials and methods (brackets denote significant differences $P<0.005$ Time 10 min Tpk1-TAP versus Tpk1-TAP kap114 $\Delta$ kap123A; $P<0.005$ Time 10 min Tpk2-TAP versus Tpk2-TAP kap108 $\Delta$ ). The mRNA expression level for ALD6 gene was determined by Northern-Blot on

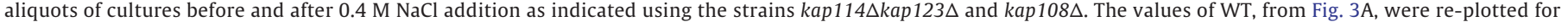
comparison. 
downregulation (Fig. 3C). Deregulated kinase activity promoted high sustained association of Snf2 and Arp8 in correlation with upregulation of RPS29B mRNA expression.

Thus the kinase activity, particularly Tpk2, regulates the remodelers association to promoters and coding regions to downregulate the gene expression during osmotic stress.

\subsection{Genomic recruitment of PKA in response to osmotic stress}

It has been previously shown that Tpk1 and Tpk2 associated with chromatin [6], particularly to the genomic loci analyzed previously, in response to oxidative stress and glucose growth. At this point our results demonstrate that in response to osmotic stress, Tpk1 translocates towards the nucleus, while Tpk2 and Bcy1 remain in their nuclear localization and that PKA is involved in the gene expression response of osmostress responsive genes. Taking into account these results, we wondered whether PKA catalytic and regulatory subunits physically associated with chromatin in response to osmotic stress in vivo.

We assessed by ChIP assays the in vivo kinetic recruitment of PKA subunits to genomic regions whose expression profile was modified by PKA activity in response to osmotic stress (Fig. 3A), namely ALD6, SED1, and HSP42 coding regions and RPS29B and RPL1B promoter regions. The coordinates corresponding to the analyzed regions are detailed in Materials and methods. Strains expressing each PKA subunit TAP-tagged from its chromosomal locus: Bcy1-TAP, Tpk1-TAP or Tpk2-TAP in a wild type or bcy1 $\Delta$ background was used in ChIP assays. Additionally, Tpk kinase-dead versions with a mutation in the active site causing inactive catalytic subunits, tpk $1^{\text {dead }}-$ TAP or tpk $2^{\text {dead }}-$ TAP mutants, were also used in order to analyze the requirement of PKA catalytic activity for chromatin association. The expression levels of each PKA subunit, analyzed by western-blot, and cell viability, assayed by spot assays, showed no change by exposure of cells to osmotic stress (Supplementary Fig. S3A and B). Fig. 4 shows Tpk and Bcy1 recruitment during osmotic stress to the ALD6, SED1, and HSP42 coding regions. In response to $\mathrm{NaCl}$ addition, Tpk1, Tpk2, and Bcy1 association reached a maximum at 10 min post-stress. In the absence of $B C Y 1$ function, an earlier and higher Tpk1 association ( $5 \mathrm{~min}$ ) than in wild type cells was observed in the three coding regions analyzed. Tpk2 showed a different recruitment behavior in HSP42 and SED1 regions, since no association was detected in the absence of $B C Y 1$. However, ALD6 coding region showed the same behavior as Tpk1 in the bcy1 $1 \Delta$ strain; that is an early and high association of Tpk2. Finally, strains expressing catalytically inactive versions of Tpk 1 or Tpk2, tpk $1^{\text {dead }}$ and $t p k 2^{\text {dead }}$, showed no association of the subunits with the chromatin under osmotic stress, indicating the requirement of catalytic activity for their association.

ChIP analysis of PKA subunits at RPS29B and RPL1B promoter regions during osmotic stress is shown in Fig. 4. Both promoter regions are mainly occupied by Tpk2 after 5 min post-osmostress. The $t p k 2^{\text {dead }}$ mutant and Bcy 1 were not found associated with chromatin in these gene regions. The deletion of Bcy1 highly affected the catalytic subunit recruitment since Tpk1 instead of Tpk2 was found associated with both ribosomal protein gene promoters in a bcy $1 \Delta$ strain.

Overall, the results indicate that in response to osmotic stress both Tpk catalytic subunits and Bcy1 regulatory subunit can be recruited to the coding regions of osmoinducible genes while only Tpk2 catalytic subunit can be recruited to promoter regions of ribosomal protein genes. The Tpk association to all gene regions assayed was completely dependent on their kinase activity. The absence of Bcy1 affected these interactions.

Finally, we studied the relationship between nuclear localization of Tpk1 and Tpk2, its association with chromatin and gene expression

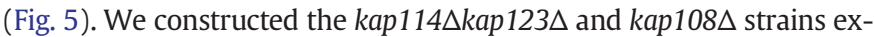
pressing Tpk1 or Tpk2 fused to the TAP tag. The strains were grown to exponential phase on YPGlu, stressed by addition of $\mathrm{NaCl}$ and assessed for Tpk recruitment to the ALD6 coding region by ChIP. In response to osmotic stress, Tpk1 was associated with an ALD6 coding region in the

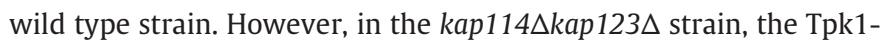
chromatin association was not detected. A similar result was obtained for Tpk2-TAP kap108 compared to the wild type strain. These results demonstrate that nuclear localization of catalytic subunits would be necessary for its association with chromatin. To test the consequence of a reduction in nuclear Tpk1 or Tpk2 accumulation on gene expression, we measured the mRNA levels of ALD6 gene in kap114 $\triangle$ kap123A or kap108 mutant strains. In kap114 1 kap123 strain ALD6 mRNA was up regulated whereas kap $108 \Delta$ strain sowed a reduced gene induction upon osmotic stress. These results suggest that, at least in a gene region analyzed, the gene expression in response to osmotic stress is dependent on the proper nuclear localization of PKA subunits and its physical interaction with chromatin.

\section{Discussion}

\subsection{Chromatin association of PKA subunits in response to osmotic stress}

In response to changes in environmental salinity, cell physiology is reprogrammed to survive in the new extracellular conditions. Osmotic responsive genes show a transient expression pattern. The transcriptional induction of most genes whose transcription responds to salt stress is dependent on the presence of the stress-activated protein kinase Hog1 [45]. PKA activity negatively regulates the efficient adaptation to osmotic stress $[41,46,47]$.

Genome wide ChIP-on-Chip experiments in S. cerevisiae revealed that Tpk1 and Tpk2 interact with chromatin, both in coding and in promoter regions under fermentative metabolism or oxidative stress [6]. We have studied in this work the kinetics of association of PKA subunits to the osmo-responsive gene regions. Our results show that both Tpk1 and Tpk2 associate with gene coding regions of ALD6, HSP42, and SED1 genes in response to osmotic stress. Surprisingly, we also found Bcy1 associated with chromatin in response to osmotic stress (Fig. 4). On promoter regions RPL1B and RPS29 of ribosomal genes, we found mainly Tpk2 association in response to osmotic stress. Unlike coding regions, no association of Bcy1 of the analyzed promoters was observed (Fig. 4). The Tpk catalytic activity is required for their association to chromatin, as occurs with protein kinases Hog1 [39] and Sty1 [5]. Deletion of $B C Y 1$ gene not only affects which catalytic subunit isoform binds to chromatin in response to osmotic stress, but also modulates the kinetics of Tpk association with chromatin (Fig. 4).

We analyzed the PKA role on gene expression of five gene regions, which are transcriptionally regulated during osmotic stress. Deletion of the regulatory subunit with the consequent constitutive activation of the cAMP-PKA pathway impairs the transient stress gene expression and therefore severely affects the cell survival under osmotic stress (Fig. 3). These results are in agreement with previous evidences showing that the adaptive stress response must be temporarily restricted, as it has been observed that constitutive activation of stress responsive pathways has a detrimental effect on cell growth. For example, sustained SAPK activation in both yeast and mammals, leads to cell cycle delay and apoptosis [48-50]. Our results suggest that the kinetics of association of Tpk1 and Tpk2 observed in wild type cells is critical for the regulation of the transient expression of osmo-responsive genes.

In wild type cells, the temporal association during osmotic stress of chromatin remodeling factors belonging to SWI/SNF and INO80 complexes, to both a coding and a promoter region, correlates with transient gene expression. We made several observations that suggest that suitable temporal association of PKA might negatively regulate the chromatin association of remodelers to efficiently promote down regulation of gene expression. First, Bcy1 negatively regulates the binding of Tpk1 and positively regulates the association of Tpk2 (Fig. 4). Second, the contribution of each Tpk catalytic isoforms in the osmostress gene expression is different; Tpk2 promotes a sustained gene expression while Tpk1 activity induces a reduction in gene expression (Fig. 3C). 
Finally, in strains with deregulated Tpk2 activity chromatin remodelers show a high and stable association to target genes (Fig. 3D).

An increased association of Hog 1 with stress-responsive genes strongly correlates with chromatin remodeling and increased gene expression [51]. Particularly, ALD6, HSP42 and SED1 are examples of genes affected by Hog 1 activity which show an increase in transcription after 5 min of stress treatment [6]. In the present work, we show that PKA associates to the same genomic regions 10-20 min after stimulation with a concomitant downregulation of their expression. In the light of our findings, we speculate that rapid and transient stressadaptive response could be regulated by primary association of the Hog1 kinase and the subsequent association of PKA. The in vivo kinetic analysis of Hog 1 and Tpk association to osmotic responsive genes needs to be examined to understand their combined mechanism of action.

\subsection{Mechanism of nuclear accumulation of PKA in response to osmotic} stress

At present, it is known that a single protein kinase distributed dynamically between cytoplasm and nucleus can regulate target gene expression by both nuclear and cytoplasmic signaling mechanisms. Consequently, nuclear-cytoplasmic kinases transport would be important to regulate differential activity in both compartments. A selective nuclearcytoplasmic transport maintains a specific composition of each compartment and offers the possibility of transient regulation of different processes. Protein nuclear transport is mediated by the interaction of the nuclear localization sequence (NLS) or nuclear export sequence (NES) of the cargoes with importin and exportin, respectively [52,53].

Subcellular localization of PKA in S. cerevisiae depends on the environmental conditions such as carbon source and stress [21,23,54]. Several post-translational modifications on regulatory and catalytic subunits of yeast PKA affect its nuclear-cytoplasmic localization [55-57]. The transport mechanism of PKA subunits through nuclear membrane has not been previously characterized. Here, we describe that the nuclear accumulation of the PKA catalytic and regulatory subunits is an active process mediated through different $\beta$-karyopherin proteins (Fig. 2). This observation suggests an individual and specific PKA subunit transport mechanism through the nuclear envelope, as has been observed for Tpk1 nuclear accumulation in response to osmotic stress (Fig. 1).

We describe that the accumulation of Tpk 1 in the nucleus was stimulated upon osmotic stress, while the nuclear localization of Tpk2 and Bcy1 showed no change (Fig. 1). A decrease in cAMP levels following osmotic stress [46] would promote an increment of Tpk1-Bcy1 interaction causing nuclear accumulation of Tpk1 in response to osmotic stress.

The in silico analysis of Tpk1, Tpk2 and Bcy1 in search of classical NLS consensus sequences [58] was unsuccessful. However, the specificity determinants of $\beta$-karyopherin for the recognition of nuclear targeting signals has recently been revised [59] indicating that it will be necessary to characterize the NLS sequences of each PKA subunit experimentally.

Our results suggest that the gene expression response to osmotic stress is dependent on the proper nuclear localization of PKA subunits and its physical interaction with chromatin (Fig. 5). However, the chromatin protein kinases association seems to be regulated by different mechanisms. For example, a chromatin association of Hog1 fused to a generic nuclear import signal (NLS) is mainly dependent on its phosphorylation more than on its nuclear accumulation [60]. Furthermore, the nuclear localization of Tor1 is apparently important for its association with rDNA promoter, however, there is an import-independent mechanism that regulates the DNA-binding activity of Tor1 [4].

Evidences indicate that signaling complexes associated with chromatin in some cases include upstream kinase, downstream effectors and their regulatory protein phosphatases $[61,62]$. The presence of the PKA holoenzyme as part of a protein complex associated with chromatin suggests a participation in the local genic regulation through the kinase activity location near the putative substrates such as transcription factors or chromatin regulators.

Supplementary data to this article can be found online at http://dx. doi.org/10.1016/j.bbagrm.2015.09.007.

\section{Transparency document}

The Transparency document associated with this article can be found, in online version.

\section{Acknowledgments}

We are grateful to S. Moreno for critical reading of manuscript and helpful comments. We are very grateful to J.C. Igual, J. Thevelein, G. Schlenstedt, and S. M. Bailer, for kindly supplying yeast strains. This work was supported by a PhD fellowship from CONICET to L. Baccarini; and by grants from Agencia Nacional de Promoción Científica y Tecnológica (PICT 2195), from the University of Buenos Aires (UBA X528), from CONICET (PIP 0519), and from Ministerio de Economía y Competitividad, Spain (BFU2011-23326). S. Rossi and P. Portela are researchers from CONICET.

\section{References}

[1] J.M. Kyriakis, J. Avruch, Mammalian mitogen-activated protein kinase signal transduction pathways activated by stress and inflammation, Physiol. Rev. 81 (2001) 807-869.

[2] M. Proft, K. Struhl, Hog1 kinase converts the Sko1-Cyc8-Tup1 repressor complex into an activator that recruits SAGA and SWI/SNF in response to osmotic stress, Mol. Cell 9 (2002) 1307-1317.

[3] E. De Nadal, M. Zapater, P.M. Alepuz, L. Sumoy, G. Mas, F. Posas, The MAPK Hog1 recruits Rpd3 histone deacetylase to activate osmoresponsive genes, Nature 427 (2004) 370-374.

[4] H. Li, C.K. Tsang, M. Watkins, P.G. Bertram, X.F. Zheng, Nutrient regulates Tor1 nuclear localization and association with rDNA promoter Nature 442 (2006) 1058-1061.

[5] A. Pascual-Ahuir, M. Proft, The Sch9 kinase is a chromatin-associated transcriptional activator of osmostress-responsive genes, EMBO J. 26 (2007) 3098-3108.

[6] D.K. Pokholok, J. Zeitlinger, N.M. Hannett, D.B. Reynolds, R.A. Young, Activated signal transduction kinases frequently occupy target genes, Science 313 (2006) 533-536.

[7] A. Schaekel, P.R. Desai, J.F. Ernst, Morphogenesis-regulated localization of protein kinase A to genomic sites in Candida albicans, BMC Genomics 14 (2013) 842.

[8] W. Reiter, S. Watt, K. Dawson, C.L. Lawrence, J. Bahler, N. Jones, C.R. Wilkinson, Fission yeast MAP kinase Sty1 is recruited to stress-induced genes, J. Biol. Chem. 283 (2008) 9945-9956.

[9] J.M. Thevelein, J.H. de Winde, Novel sensing mechanisms and targets for the cAMPprotein kinase A pathway in the yeast Saccharomyces cerevisiae, Mol. Microbiol. 33 (1999) 904-918.

[10] T. Toda, S. Cameron, P. Sass, M. Zoller, J.D. Scott, B. McMullen, M. Hurwitz, E.G. Krebs, $\mathrm{M}$. Wigler, Cloning and characterization of BCY1, a locus encoding a regulatory subunit of the cyclic AMP-dependent protein kinase in Saccharomyces cerevisiae, Mol. Cell. Biol. 7 (1987) 1371-1377.

[11] T. Toda, S. Cameron, P. Sass, M. Zoller, M. Wigler, Three different genes in S. cerevisiae encode the catalytic subunits of the cAMP-dependent protein kinase, Cell 50 (1987) 277-287.

[12] I. Pedruzzi, N. Burckert, P. Egger, C. De Virgilio, Saccharomyces cerevisiaeRas/cAMP pathway controls post-diauxic shift element-dependent transcription through the zinc finger protein Gis1, EMBO J. 19 (2000) 2569-2579.

[13] M.T. Martinez-Pastor, G. Marchler, C. Schuller, A. Marchler-Bauer, H. Ruis, F. Estruch, The Saccharomyces cerevisiae zinc finger proteins Msn2p and Msn4p are required for transcriptional induction through the stress response element (STRE), EMBO J. 15 (1996) 2227-2235.

[14] W. Gorner, E. Durchschlag, M.T. Martinez-Pastor, F. Estruch, G. Ammerer, B. Hamilton, H. Ruis, C. Schuller, Nuclear localization of the $\mathrm{C} 2 \mathrm{H} 2$ zinc finger protein Msn2p is regulated by stress and protein kinase A activity, Genes Dev. 12 (1998) 586-597.

[15] Y.W. Chang, S.C. Howard, P.K. Herman, The Ras/PKA signaling pathway directly targets the Srb9 protein, a component of the general RNA polymerase II transcription apparatus, Mol. Cell 15 (2004) 107-116.

[16] V. Pelechano, S. Jimeno-Gonzalez, A. Rodriguez-Gil, J. Garcia-Martinez, J.E. PerezOrtin, S. Chavez, Regulon-specific control of transcription elongation across the yeast genome, PLoS Genet. 5 (2009) e1000614

[17] A. Roy, Y.J. Shin, K.H. Cho, J.H. Kim, Mth1 regulates the interaction between the Rgt1 repressor and the Ssn6-Tup1 corepressor complex by modulating PKA-dependent phosphorylation of Rgt1, Mol. Biol. Cell 24 (2013) 1493-1503.

[18] L.S. Robertson, G.R. Fink, The three yeast a kinases have specific signaling functions in pseudohyphal growth, Proc. Natl. Acad. Sci. U. S. A. 95 (1998) 13783-13787.

[19] X. Pan, J. Heitman, Cyclic AMP-dependent protein kinase regulates pseudohyphal differentiation in Saccharomyces cerevisiae, Mol. Cell. Biol. 19 (1999) 4874-4887. 
[20] M. Malcher, S. Schladebeck, H.U. Mosch, The Yak1 protein kinase lies at the center of a regulatory cascade affecting adhesive growth and stress resistance in Saccharomyces cerevisiae, Genetics 187 (2011) 717-730.

[21] V. Tudisca, V. Recouvreux, S. Moreno, E. Boy-Marcotte, M. Jacquet, P. Portela, Differential localization to cytoplasm, nucleus or P-bodies of yeast PKA subunits under different growth conditions, Eur. J. Cell Biol. 89 (2010) 339-348.

[22] G. Griffioen, P. Anghileri, E. Imre, M.D. Baroni, H. Ruis, Nutritional control of nucleocytoplasmic localization of cAMP-dependent protein kinase catalytic and regulatory subunits in Saccharomyces cerevisiae, J. Biol. Chem. 275 (2000) 1449-1456.

[23] V. Tudisca, C. Simpson, L. Castelli, J. Lui, N. Hoyle, S. Moreno, M. Ashe, P. Portela, PKA isoforms coordinate mRNA fate during nutrient starvation, J. Cell Sci. 125 (2012) 5221-5232.

[24] D.M. Leslie, W. Zhang, B.L. Timney, B.T. Chait, M.P. Rout, R.W. Wozniak, J.D. Aitchison, Characterization of karyopherin cargoes reveals unique mechanisms of Kap121p-mediated nuclear import, Mol. Cell. Biol. 24 (2004) 8487-8503.

[25] A. Bakhrat, K. Baranes, O. Krichevsky, I. Rom, G. Schlenstedt, S. Pietrokovski, D. Raveh, Nuclear import of ho endonuclease utilizes two nuclear localization signals and four importins of the ribosomal import system, J. Biol. Chem. 281 (2006) 12218-12226.

[26] S. Caesar, M. Greiner, G. Schlenstedt, Kap120 functions as a nuclear import receptor for ribosome assembly factor Rpf1 in yeast, Mol. Cell. Biol. 26 (2006) 3170-3180.

[27] N. Mosammaparast, Y. Guo, J. Shabanowitz, D.F. Hunt, L.F. Pemberton, Pathways mediating the nuclear import of histones H3 and H4 in yeast, J. Biol. Chem. 277 (2002) 862-868.

[28] M. Greiner, S. Caesar, G. Schlenstedt, The histones H2A/H2B and H3/H4 are imported into the yeast nucleus by different mechanisms, Eur. J. Cell Biol. 83 (2004) 511-520.

[29] X. Quan, J. Yu, H. Bussey, U. Stochaj, The localization of nuclear exporters of the importin-beta family is regulated by Snf1 kinase, nutrient supply and stress, Biochim. Biophys. Acta 1773 (2007) 1052-1061.

[30] U. Stochaj, R. Rassadi, J. Chiu, Stress-mediated inhibition of the classical nuclear protein import pathway and nuclear accumulation of the small GTPase Gsp1p, FASEB J. 14 (2000) 2130-2132.

[31] C.M. Demlow, T.D. Fox, Activity of mitochondrially synthesized reporter proteins is lower than that of imported proteins and is increased by lowering cAMP in glucose-grown Saccharomyces cerevisiae cells, Genetics 165 (2003) 961-974.

[32] H. Ito, Y. Fukuda, K. Murata, A. Kimura, Transformation of intact yeast cells treated with alkali cations, J. Bacteriol. 153 (1983) 163-168.

[33] W.K. Huh, J.V. Falvo, L.C. Gerke, A.S. Carroll, R.W. Howson, J.S. Weissman, E.K. O'Shea, Global analysis of protein localization in budding yeast, Nature 425 (2003) 686-691.

[34] N. Shulga, N. Mosammaparast, R. Wozniak, D.S. Goldfarb, Yeast nucleoporins involved in passive nuclear envelope permeability, J. Cell Biol. 149 (2000) 1027-1038.

[35] P.M. Roberts, D.S. Goldfarb, In vivo nuclear transport kinetics in Saccharomyces cerevisiae, Methods Cell Biol. 53 (1998) 545-557.

[36] L. Kuras, K. Struhl, Binding of TBP to promoters in vivo is stimulated by activators and requires Pol II holoenzyme, Nature 399 (1999) 609-613.

[37] M. Proft, A. Pascual-Ahuir, E. de Nadal, J. Arino, R. Serrano, F. Posas, Regulation of the Sko1 transcriptional repressor by the Hog1 MAP kinase in response to osmotic stress, EMBO J. 20 (2001) 1123-1133.

[38] B. Martinez-Bono, I. Quilis, E. Zalve, J.C. Igual, Yeast karyopherins Kap123 and Kap95 are related to the function of the cell integrity pathway, FEMS Yeast Res. 10 (2010) 28-37.

[39] D.B. Berry, A.P. Gasch, Stress-activated genomic expression changes serve a preparative role for impending stress in yeast, Mol. Biol. Cell 19 (2008) 4580-4587.

[40] H.C. Causton, B. Ren, S.S. Koh, C.T. Harbison, E. Kanin, E.G. Jennings, T.I. Lee, H.L. True, E.S. Lander, R.A. Young, Remodeling of yeast genome expression in response to environmental changes, Mol. Biol. Cell 12 (2001) 323-337.

[41] J. Norbeck, A. Blomberg, The level of cAMP-dependent protein kinase A activity strongly affects osmotolerance and osmo-instigated gene expression changes in Saccharomyces cerevisiae, Yeast 16 (2000) 121-137.
[42] A. Saha, J. Wittmeyer, B.R. Cairns, Chromatin remodeling by RSC involves ATPdependent DNA translocation, Genes Dev. 16 (2002) 2120-2134.

[43] J. Ford, O. Odeyale, C.H. Shen, Activator-dependent recruitment of SWI/SNF and INO80 during INO1 activation, Biochem. Biophys. Res. Commun. 373 (2008) 602-606.

[44] E. Klopf, L. Paskova, C. Sole, G. Mas, A. Petryshyn, F. Posas, U. Wintersberger, G. Ammerer, C. Schuller, Cooperation between the INO80 complex and histone chaperones determines adaptation of stress gene transcription in the yeast Saccharomyces cerevisiae, Mol. Cell. Biol. 29 (2009) 4994-5007.

[45] F. Posas, J.R. Chambers, J.A. Heyman, J.P. Hoeffler, E. de Nadal, J. Arino, The transcriptional response of yeast to saline stress, J. Biol. Chem. 275 (2000) 17249-17255.

[46] J.A. Marquez, R. Serrano, Multiple transduction pathways regulate the sodiumextrusion gene PMR2/ENA1 during salt stress in yeast, FEBS Lett. 382 (1996) 89-92.

[47] A. Pascual-Ahuir, F. Posas, R. Serrano, M. Proft, Multiple levels of control regulate the yeast cAMP-response element-binding protein repressor Sko1p in response to stress, J. Biol. Chem. 276 (2001) 37373-37378.

[48] G. Yaakov, M. Bell, S. Hohmann, D. Engelberg, Combination of two activating mutations in one HOG1 gene forms hyperactive enzymes that induce growth arrest, Mol. Cell. Biol. 23 (2003) 4826-4840

[49] A. Vendrell, M. Martinez-Pastor, A. Gonzalez-Novo, A. Pascual-Ahuir, D.A. Sinclair, M. Proft, F. Posas, Sir2 histone deacetylase prevents programmed cell death caused by sustained activation of the Hog1 stress-activated protein kinase, EMBO Rep. 12 (2011) 1062-1068

[50] I. Dolado, A.R. Nebreda, AKT and oxidative stress team up to kill cancer cells, Cancer Cell 14 (2008) 427-429.

[51] E. de Nadal, F. Posas, Osmostress-induced gene expression - a model to understand how stress-activated protein kinases (SAPKs) regulate transcription, FEBS J. (2015).

[52] A.C. Strom, K. Weis, Importin-beta-like nuclear transport receptors, Genome Biol. 2 (2001) (REVIEWS3008)

[53] K. Weis, Nucleocytoplasmic transport: cargo trafficking across the border, Curr. Opin. Cell Biol. 14 (2002) 328-335.

[54] G. Griffioen, S. Swinnen, J.M. Thevelein, Feedback inhibition on cell wall integrity signaling by Zds1 involves Gsk3 phosphorylation of a cAMP-dependent protein kinase regulatory subunit, J. Biol. Chem. 278 (2003) 23460-23471.

[55] C.A. Solari, V. Tudisca, M. Pugliessi, A.D. Nadra, S. Moreno, P. Portela, Regulation of PKA activity by an autophosphorylation mechanism in Saccharomyces cerevisiae, Biochem. J. 462 (2014) 567-579.

[56] S. Haesendonckx, V. Tudisca, K. Voordeckers, S. Moreno, J.M. Thevelein, P. Portela, The activation loop of PKA catalytic isoforms is differentially phosphorylated by Pkh protein kinases in Saccharomyces cerevisiae, Biochem. J. 448 (2012) 307-320.

[57] A. Soulard, A. Cremonesi, S. Moes, F. Schutz, P. Jeno, M.N. Hall, The rapamycinsensitive phosphoproteome reveals that TOR controls protein kinase A toward some but not all substrates, Mol. Biol. Cell 21 (2010) 3475-3486.

[58] S. Kosugi, M. Hasebe, M. Tomita, H. Yanagawa, Systematic identification of cell cycledependent yeast nucleocytoplasmic shuttling proteins by prediction of composite motifs, Proc. Natl. Acad. Sci. U. S. A. 106 (2009) 10171-10176.

[59] Y.M. Chook, K.E. Suel, Nuclear import by karyopherin-betas: recognition and inhibition, Biochim. Biophys. Acta 1813 (2011) 1593-1606.

[60] P.M. Alepuz, A. Jovanovic, V. Reiser, G. Ammerer, Stress-induced map kinase Hog1 is part of transcription activation complexes, Mol. Cell 7 (2001) 767-777.

[61] J.D. Nelson, R.C. LeBoeuf, K. Bomsztyk, Direct recruitment of insulin receptor and ERK signaling cascade to insulin-inducible gene loci, Diabetes 60 (2011) 127-137.

[62] M.C. Lawrence, C. Shao, K. McGlynn, B. Naziruddin, M.F. Levy, M.H. Cobb, Multiple chromatin-bound protein kinases assemble factors that regulate insulin gene transcription, Proc. Natl. Acad. Sci. U. S. A. 106 (2009) 22181-22186. 